\title{
High Circular Polarization of Electroluminescence Achieved via Self- Assembly of a Light-Emitting Chiral Conjugated Polymer into Multidomain Cholesteric Films
}

\author{
Daniele Di Nuzzo, ${ }^{*}{ }^{\dagger}$ Chidambar Kulkarni, ${ }^{\ddagger}$ Baodan Zhao $^{\dagger}$ Eilam Smolinsky, ${ }^{\S}$ Francesco Tassinari, ${ }^{\S}$ \\ Stefan C. J. Meskers, ${ }^{\dagger \odot}$ Ron Naaman, ${ }^{\S \odot}$ E. W. Meijer, ${ }^{\ddagger \odot}$ and Richard H. Friend ${ }^{\dagger \oplus}$ \\ ${ }^{\dagger}$ Cavendish Laboratory, University of Cambridge, JJ Thomson Avenue, Cambridge, CB3 OHE, United Kingdom \\ ${ }^{\ddagger}$ Institute for Complex Molecular Systems, Laboratory of Macromolecular and Organic Chemistry, Eindhoven University of \\ Technology, PO Box 513, Eindhoven, 5600 MB, The Netherlands \\ ${ }^{\S}$ Department of Chemical Physics, Weizmann Institute of Science, Rehovot 76100, Israel
}

\section{Supporting Information}

ABSTRACT: We demonstrate a facile route to obtain high and broad-band circular polarization of electroluminescence in single-layer polymer OLEDs. As a light-emitting material we use a donor-acceptor polyfluorene with enantiomerically pure chiral side-chains. We show that upon thermal annealing the polymer self-assembles into a multidomain cholesteric film. By varying the thickness of the polymer emitting layer, we achieve high levels of circular polarization of electroluminescence (up to $40 \%$ excess of right-handed polarization), which are the highest reported for polymer

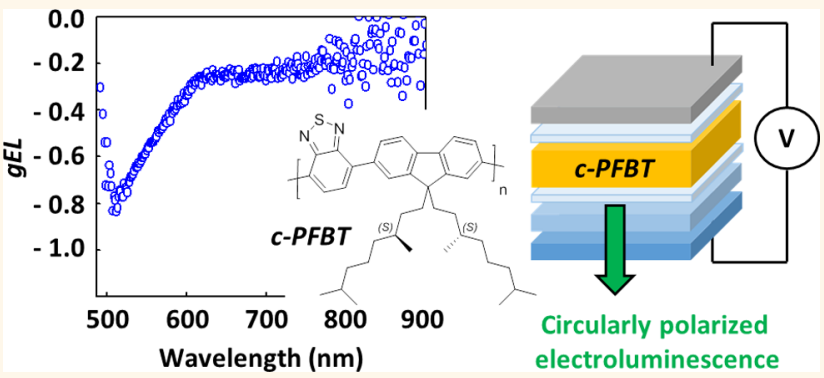
OLEDs not using chiral dopants or alignment layers. Mueller matrix ellipsometry shows strong optical anisotropies in the film, indicating that the circular polarization of luminescence arises mainly after the photon has been generated, through selective scattering and birefringence correlated in the direction of the initial linear polarization of the photon. Our work demonstrates that chirally substituted conjugated polymers can combine photonic and semiconducting properties in advanced optoelectronic devices.

KEYWORDS: OLEDs, circularly polarized luminescence, light-emitting polymers, chirality, self-assembly

S ources of circularly polarized light are interesting for a range of applications across different fields such as spinoptoelectronics, ${ }^{1,2}$ optical manipulation of information, ${ }^{3,4}$ 3D imaging systems, biology, and medicine. ${ }^{5-7}$ Organic LEDs can emit circularly polarized electroluminescence (CPEL) when the emitting organic material is chiral and of one handedness only. This makes them excellent candidates as sources of circularly polarized light, due to the ease of fabrication, possible large-area processing, low cost, and freedom to tune the optoelectronic properties of the organic semiconductor via molecular design.

Moreover, CPEL is highly desirable to enhance the efficiency of OLED-based displays: to enhance the contrast of OLEDbased commercial displays, circularly polarizing filters are applied that trap ambient light in the display. This implies that, because it is randomly polarized, only half of the electro- luminescence (EL) can exit the display. Attaining full circular polarization of EL could therefore double the efficiency of OLED-based displays.

In recent years, considerable efforts have been made to achieve strong CPEL in OLEDs. However, besides two recent examples $^{8,9}$ low levels of polarization are commonly achieved, below $10-15 \%$ excess of one handedness (Table S1, Supporting Information). Several types of materials for the emitting layer have been used: lanthanide complexes, ${ }^{9}$ phosphorescent organic complexes, ${ }^{10-12}$ and conjugated polymers. ${ }^{8,13-16}$ It is useful to point out that two main distinct mechanisms can generate circularly polarized luminescence

Received: October 18, 2017

Accepted: November 28, 2017

Published: November 28, 2017 
a)

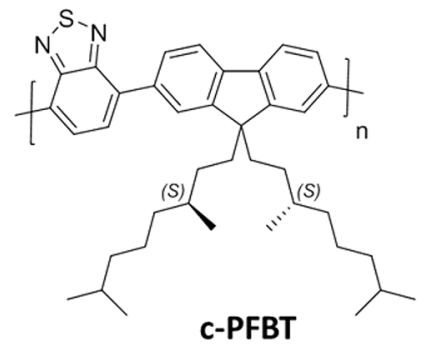

b)
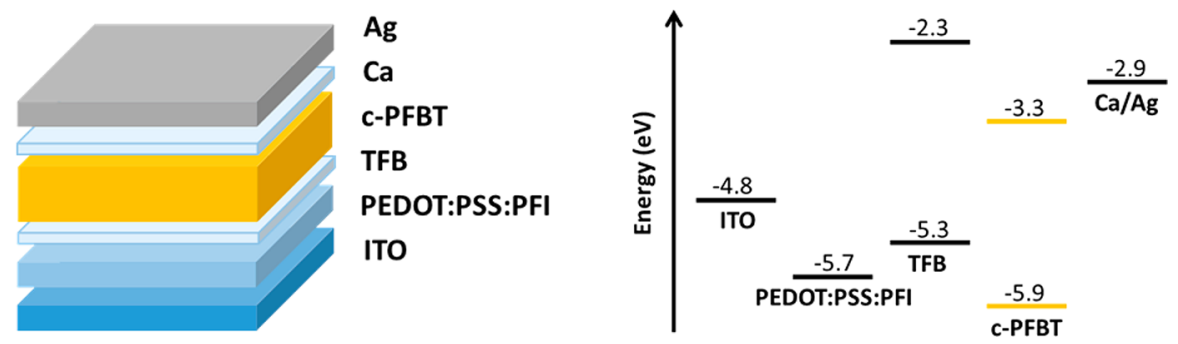

Figure 1. (a) Chemical structure of c-PFBT. (b) Layers comprising the OLED stack and their energy levels.

(CPL) in molecular materials. First, CPL can originate locally, i.e., at the molecular site of light emission. In this case, for the emitted light to be fully circularly polarized, the optical transition has to be magnetic-dipole allowed and electric-dipole forbidden. ${ }^{17}$ This requirement poses a challenge for reaching strong circular polarization while maintaining high luminescence efficiency. To date, the only known examples of chiral molecules with high luminescence efficiency and high circular polarization are chiral lanthanide complexes. ${ }^{18}$ The second mechanism that can be exploited to obtain CPL is nonlocal: this is the case of luminescent cholesteric liquid crystals, for which, in the limit of thick films $\left(10^{2} \mathrm{~nm}\right)$, circular polarization is largely determined by the anisotropy of the cholesteric dielectric medium and is weakly dependent on the polarization state of light at the site of emission. Strong CPL in polymer films $^{19}$ and CPEL in polymer OLEDs ${ }^{8,15}$ were achieved by exploiting this mechanism: in these works alignment layers were used to induce the formation of large cholesteric domains. In some recent cases, chirality was obtained by mixing achiral polymers with chiral dopants. 8,16

In this paper we exploit the nonlocal effect of cholesteric order to achieve high levels of CPEL in OLEDs based on a chirally substituted polyfluorene copolymer with $100 \%$ enantiomeric purity acting as emitting layer, without using any chiral dopant and alignment layers. By varying the thickness of the emitting layer, we achieve CPEL with up to $40 \%$ excess of right-handed polarization, the second highest value for polymer OLEDs and the highest obtained without the use of chiral dopants or alignment layers. Our polymer consists of a fluorene-benzothiadiazole donor-acceptor repeat unit forming the conjugated backbone, with chiral centers attached to the fluorene moiety to provide enhanced solubility and chiral ordering with preferred handedness in the assembled state. Consistent with previous studies, we observe that self-assembly on the mesoscale occurs when the polymer films are thermally annealed into the liquid crystal phase, giving rise to cholesteric order. $^{20,21}$ Furthermore, we find that the annealed films have multidomain cholesteric liquid crystalline order, with domains on the order of a few hundreds of nanometers in size. We interpret the observed CPEL as arising from circular selective scattering and birefringence in such multidomain films. Our results show that OLEDs based on multidomain cholesteric emitting layers allow strong and broad-band CPEL.

\section{RESULTS AND DISCUSSION}

In Figure 1 the chemical structure of chiral and enantiomerically pure poly(fluorene-alt-benzothiadiazole) (c-PFBT) is shown. c-PFBT is used as emitting layer in the OLEDs presented in this work. A standard OLED structure was used: the layers and their respective energy levels are shown in Figure 1. Hole injection is achieved via a layer of PEDOT:PSS:PFI deposited on top of ITO. PFI (perfluorinated ionomer, see Methods for details) was added to increase the work function of PEDOT:PSS and reduce the energy barrier for hole injection into c-PFBT. ${ }^{22}$ OLEDs with different thicknesses of the cPFBT emitting layer were fabricated; the thickness of the emitting layer was varied by varying the concentration of cPFBT in the solution for spin coating, while fixed thicknesses were used for all the other layers. After spin-coating c-PFBT, the samples were annealed at $240{ }^{\circ} \mathrm{C}$ for $15 \mathrm{~min}$ in a nitrogen atmosphere. An evaporated top contact consisting of calcium $(20 \mathrm{~nm})$ and silver $(80 \mathrm{~nm})$ was used for electron injection. A thin layer of poly[2,7-9,9-di- $n$-octylfluorene-alt-1,4-phenylene4-sec-butylphenylimino-1,4-phenylene] (TFB) was deposited between PEDOT:PSS:PFI and c-PFBT to act as electronblocker. $^{23}$

The dissymmetry factor $g_{\mathrm{EL}}$ in the electroluminescence of OLEDs comprising a c-PFBT layer of 100, 200, and $400 \mathrm{~nm}$ thickness, operated in continuous voltage bias at 10,15 , and 25 $\mathrm{V}$, respectively, is shown in Figure 2a. This corresponded to current densities of 340,565 , and $13 \mathrm{~mA} / \mathrm{cm}^{2}$ and luminances of 900,2950 , and $80 \mathrm{~cd} / \mathrm{m}^{2}$, respectively. The currentluminance-voltage characterization of the OLEDs is shown in Figure $\mathrm{S} 1$. We define here the EL dissymmetry factor $g_{\mathrm{EL}}$ as

$$
g_{\mathrm{EL}}=2\left[\mathrm{EL}_{\mathrm{L}}-\mathrm{EL}_{\mathrm{R}}\right] /\left[\mathrm{EL}_{\mathrm{L}}+\mathrm{EL}_{\mathrm{R}}\right]
$$



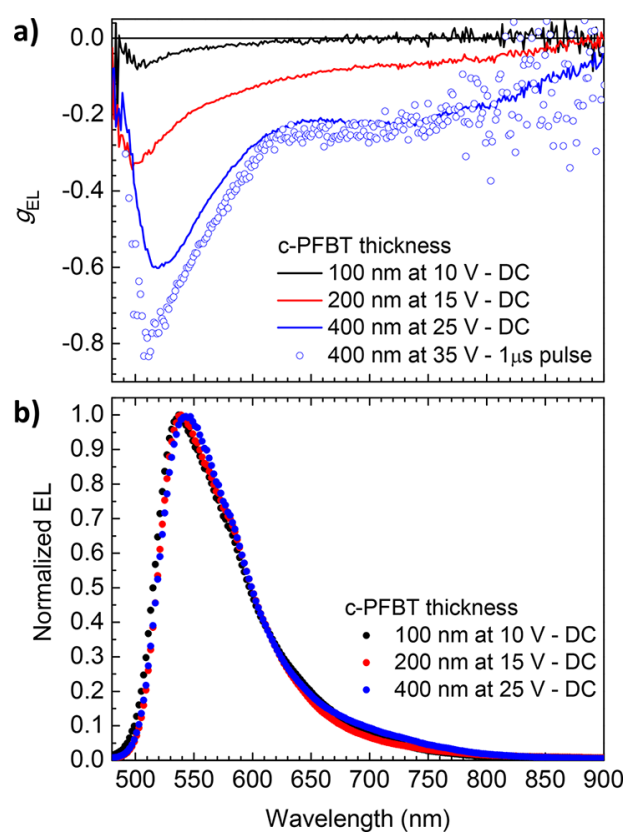

Figure 2. (a) Dissymmetry factor of electroluminescence $g_{\mathrm{EL}}$ of OLEDs comprising a c-PFBT emitting layer of varying thickness (see legend). $g_{\mathrm{EL}}$ was measured at constant voltage bias ("DC", solid lines). $g_{\mathrm{EL}}$ measured under $1 \mu \mathrm{s}, 35 \mathrm{~V}$ pulsed-voltage excitation (1 $\mathrm{kHz}$ repetition rate) on the $400 \mathrm{~nm}$ thick OLED is also shown (circles). (b) Corresponding total, unpolarized EL spectra under constant voltage bias.

where $E L_{L}$ and $E L_{R}$ are the EL intensities with left-handed and right-handed circular polarization, respectively. The setup used to measure $\mathrm{EL}_{\mathrm{L}}$ and $\mathrm{EL}_{\mathrm{R}}$ is described in the Methods section. We measured an excess of right-handed circular polarization in the EL of all the OLEDs, giving negative values of $g_{\mathrm{EL}}$ according to the definition above. By increasing the thickness of the cPFBT emitting layer, $g_{\mathrm{EL}}$ changes dramatically: with a c-PFBT layer thickness of $100 \mathrm{~nm}$ we observe that $g_{\mathrm{EL}}$ reaches a minimum value of -0.08 at $500 \mathrm{~nm}$. On increasing the thickness of the c-PFBT layer to $200 \mathrm{~nm}, g_{\mathrm{EL}}$ reaches -0.33 at $500 \mathrm{~nm}$. We note that this is in line with the values of $g_{\mathrm{EL}}$ reported for most circularly polarized OLEDs to date (Supporting Table S1). When we further increase the thickness of the c-PFBT layer to $400 \mathrm{~nm}$, we obtain a minimum $g_{\mathrm{EL}}$ of -0.6 , with the peak wavelength shifting to $520 \mathrm{~nm}$. Not only the minimum value, but also the dependence of $g_{\mathrm{EL}}$ on wavelength varies with the thickness of the c-PFBT layer: with the $400 \mathrm{~nm}$ thick OLED a band centered at 700-750 nm appears, as opposed to the thinner devices, where $g_{\mathrm{EL}}$ decreases monotonically with wavelength.

We have also measured CPEL under pulsed-voltage operation of the OLEDs: Figure $2 \mathrm{a}$ shows $g_{\mathrm{EL}}$ measured on the $400 \mathrm{~nm}$ thick OLED when applying square voltage pulses (going from 0 to $35 \mathrm{~V}$ ) with a duration of $1 \mu \mathrm{s}$, at a repetition rate of $1 \mathrm{kHz}$; we observe that CPEL is higher than in the continuous bias case, with $g_{\mathrm{EL}}$ reaching -0.8 at $510 \mathrm{~nm}$ (i.e., corresponding to $40 \%$ excess of right-handed polarization). We found that $g_{\mathrm{EL}}$ decreases when the duration of the pulses is increased, reaching a less negative value of -0.65 at $525 \mathrm{~nm}$ under DC bias; furthermore, the peak of $g_{\mathrm{EL}}$ shifts to longer wavelengths for the longest pulses $(250 \mu \mathrm{s})$, reaching $525 \mathrm{~nm}$ for the constant voltage case (Figure S2, Supporting Information). The total, unpolarized EL of the same OLEDs is shown in Figure 2b. We observe a red-shift of EL when going from 100 and $200 \mathrm{~nm}$ c-PFBT layers to $400 \mathrm{~nm}$, with the peak of EL shifting from $535 \mathrm{~nm}$ to $545 \mathrm{~nm}$. We interpret the changes in EL spectrum as originating from different outcoupling of light when the c-PFBT layer thickness is varied.

All the CPEL results presented above were obtained on OLEDs in which the c-PFBT layer had been thermally annealed at $240{ }^{\circ} \mathrm{C}$ before evaporating the top contact. No CPEL was detected on as-cast OLEDs. The dependence on annealing temperature and thickness of the chiroptical properties of films of chirally substituted polyfluorenes is known: it was previously shown that c-PFBT enters the cholesteric liquid crystalline phase when heated above $150{ }^{\circ} \mathrm{C} .^{20}$ Furthermore, it was shown on chiral polyfluorenes that the circular polarization dissymmetry of light transmitted through annealed films is strongly dependent on film thickness, owing to the longrange, nonlocal effect of the cholesteric dielectric on light polarization. ${ }^{21}$ Consistently with these previous studies, we observe a 25-fold increase in apparent circular dichroism upon thermal annealing of thin films of c-PFBT (Figure 3a). The internal structural organization of the film leads to unusual optical characteristics, and we need therefore to take great care in the definition of the experimental quantities under investigation. In Figure $3 a$ we show the difference in extinction $E=-\log _{10}(T)$ between transmission $(T)$ of incident light having left and right circular polarization and using unpolarized $(U)$ detection. The resulting quantity $E_{\mathrm{L}}{ }^{\mathrm{U}}-E_{\mathrm{R}}^{\mathrm{U}}$ can be regarded as an apparent circular dichroism where we note that in principle both absorption and scattering can contribute to extinction. In the wavelength region above $500 \mathrm{~nm}$, where the polymer does not show allowed optical absorption, the circular differential extinction is mainly due to circular selective scattering of light.

Figure $3 \mathrm{~b}$ and $\mathrm{c}$ show atomic force microscopy (AFM) images of as-cast and annealed $400 \mathrm{~nm}$ thick films deposited on fused silica substrates, respectively. As-cast films show spherical morphology (Figure 3b), whereas upon annealing the morphology changes to an interconnected network of fibers (Figure 3c). The fibers are typically ca. 200-300 nm wide. Polarized optical microscopy (POM) with c-PFBT films between two crossed polarizers shows no birefringence for ascast films (Figure 3d). In contrast, the annealed films do show birefringence, without any preferred orientation (Figure 3e). The image of the annealed film suggests small birefringent domains with dimensions on the order of $1 \mu \mathrm{m}$. The strong dissymmetry in extinction and the presence of nonoriented birefringence in POM indicate that upon thermal annealing multidomain cholesteric films are produced, containing cholesteric domains oriented at different angles between each other. Note that we could not observe macroscopic circular selective reflection on the films; this observation confirms the disordered nature of the films. ${ }^{24}$

The high degree of circular polarization in electroluminescence (Figure 2) and its relation to the molecular organization induced by thermal annealing (Figure 3) are intriguing and will now be discussed in more detail. In Figure $4 a$ we show the chiroptical properties of an annealed film of c-PFBT spin coated with the same thickness as used in the electroluminescence measurements $(400 \mathrm{~nm})$. We find very high values for the dissymmetry ratio in extinction defined as $g_{\mathrm{EXT}}=$ $2\left[E_{\mathrm{L}}^{\mathrm{U}}-E_{\mathrm{R}}^{\mathrm{U}}\right] /\left[E_{\mathrm{L}}^{\mathrm{U}}+E_{\mathrm{R}}^{\mathrm{U}}\right]$. Near the onset of the absorption we find $g_{\mathrm{EXT}}=-0.8$. The dissymmetry ratio for photoluminescence, $g_{\mathrm{PL}}=2\left[I_{\mathrm{L}}-I_{\mathrm{R}}\right] /\left[I_{\mathrm{L}}+I_{\mathrm{R}}\right]$, with $I_{\mathrm{L}}$ and $I_{\mathrm{R}}$ being 
a)
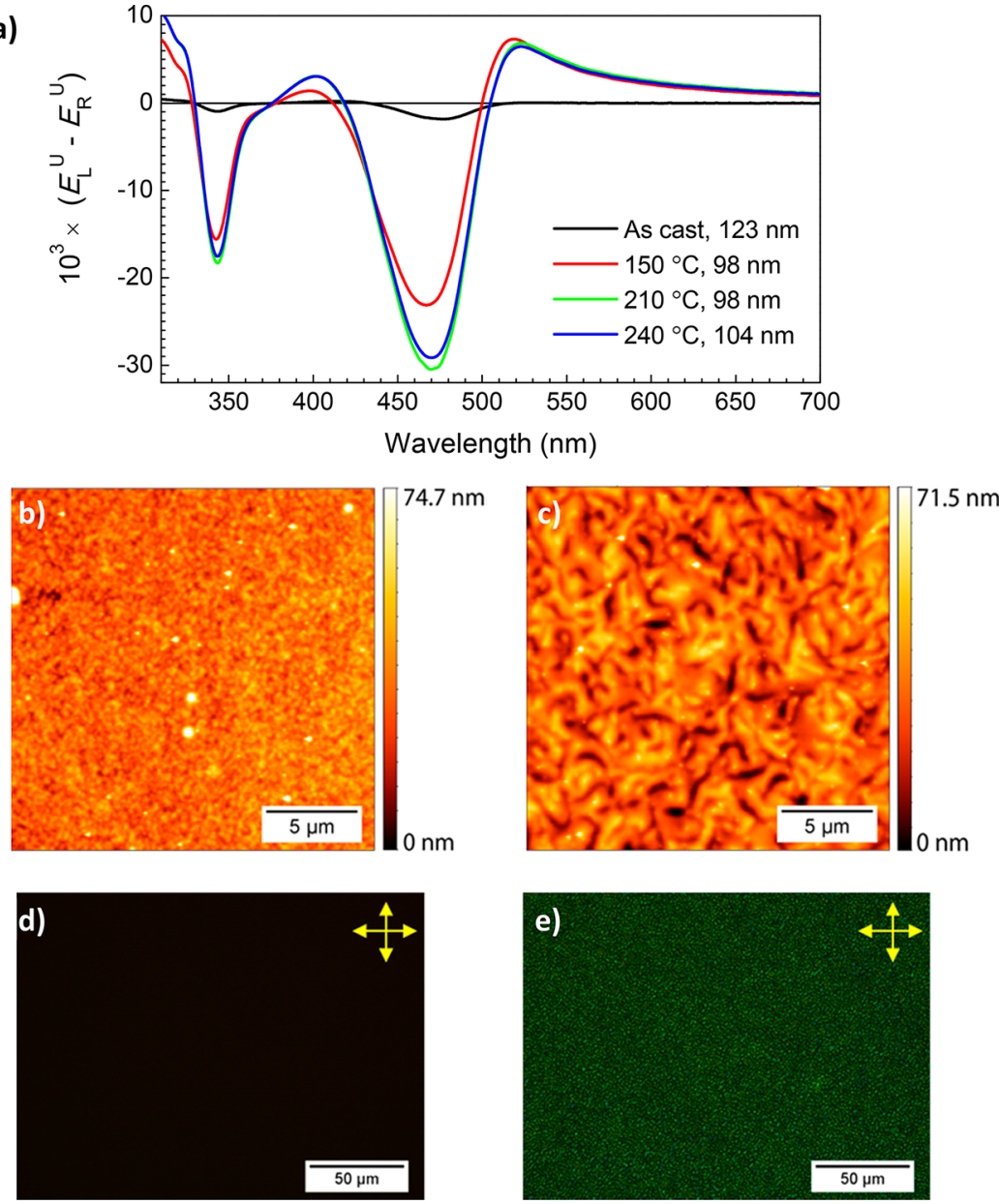

Figure 3. (a) Difference in extinction $E$ for left and right circularly polarized incident light and unpolarized detection, for films of c-PFBT with thickness around $100 \mathrm{~nm}$, before and after annealing at different temperatures (see legend). (b, c) Tapping-mode AFM height images of ascast and annealed films, respectively. (d, e) Polarized optical microscopy (POM) images of as-cast and annealed films, respectively, when viewed under a cross-polarizer. All the films were deposited on fused silica substrates. The thermal annealing of films for AFM and POM was performed at $240{ }^{\circ} \mathrm{C}$ for $15 \mathrm{~min}$.

the intensity of left and right circularly polarized luminescence for the same $400 \mathrm{~nm}$ thick film, also reaches high values near the onset of the luminescence: $g_{\mathrm{PL}}=-0.6$. The photoluminescence has been measured using unpolarized excitation light with $356 \mathrm{~nm}$ wavelength and with direction normal to the film and in line with the direction of luminescence collection. The similarity between $g_{\mathrm{EXT}}$ and $g_{\mathrm{PL}}$ at the onsets of the absorption and luminescence bands is consistent with the expected time reversal symmetry for photon absorption and emission near the origin of the bands. The high values for $g_{\mathrm{EL}}$ $g_{\mathrm{EXT}}$, and $g_{\mathrm{PL}}$ are all consistent with a nonlocal origin of the circular polarization related to a cholesteric organization within the film.

The next issue we address is how the peculiar multidomain cholesteric organization in the films under study can lead to such high degrees of polarization in photo- and electroluminescence. The hypothesis we investigate is that the light produced by electroluminescence acquires its polarization characteristics while it travels through the film in the outward direction, after the corresponding photon has been generated. In order to test this hypothesis, we investigated the transmission of light in the wavelength range corresponding to the emission. In a first naive approach, we assume that initially the emission is unpolarized and that the relative circular differential transmission of the left and right circular components of the emission leads to net circular polarization. In Figure $4 \mathrm{~b}$ we illustrate the dissymmetry ratio $g_{\text {TRANS }}=2\left[T_{\mathrm{L}}^{\mathrm{U}}-T_{\mathrm{R}}^{\mathrm{U}}\right] /\left[T_{\mathrm{L}}^{\mathrm{U}}+\right.$ $\left.T_{\mathrm{R}}{ }^{\mathrm{U}}\right]$ for transmission of left and right circularly polarized incident light and unpolarized detection for thermally annealed films of c-PFBT with different thicknesses. For the thickest film (400 nm), a broad band in the $g_{\text {TRANS }}$ spectrum appears for wavelengths near $1100 \mathrm{~nm}$ (see inset in Figure 4b). Because the polymer has no absorption band in this region and does not show any detectable circular selective reflection of light, we assign the negative $g_{\text {TRANS }}$ to preferential scattering of left circularly polarized light. The sign and wavelength are consistent with a left-handed cholesteric organization with pitch length around $600 \mathrm{~nm}$ and refractive index near 1.7. In the wavelength range 490-700 $\mathrm{nm}$ relevant for the emission, the degree of polarization is on the order of $10^{-2}$ (preferred right-handed transmission). These results show that selective scattering can at least partially contribute to the circular polarization of EL observed in the OLEDs.

A second nonlocal effect that can impact the CPEL in our OLEDs is birefringence. Using a rubbed polyimide alignment layer as substrate for thin thermally annealed films of c-PFBT, we found that the absorption of light involving the allowed transition to the lowest singlet excited state $\left(S_{1}\right)$ is highly 

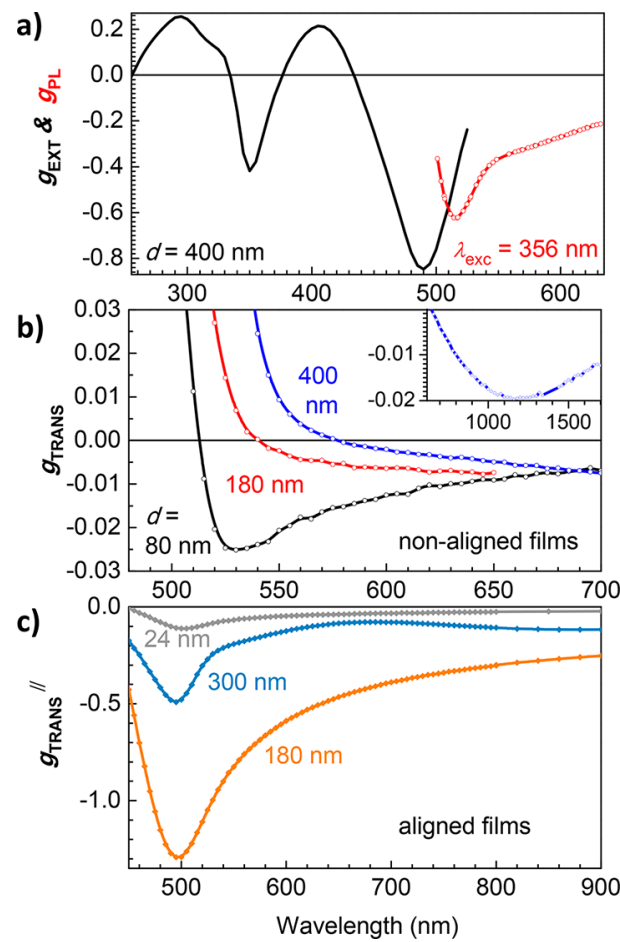

Figure 4. (a) Dissymmetry ratio $g_{\mathrm{EXT}}=2\left(E_{\mathrm{L}}^{\mathrm{U}}-E_{\mathrm{R}}^{\mathrm{U}}\right) /\left(E_{\mathrm{L}}^{\mathrm{U}}+E_{\mathrm{R}}^{\mathrm{U}}\right)$ for extinction of left and right circularly polarized incident light and unpolarized detection on a $400 \mathrm{~nm}$ thick, thermally annealed film of c-PFBT on fused silica. In red, the dissymmetry ratio for photoluminescence, $g_{\mathrm{PL}}=2\left[I_{\mathrm{L}}-I_{\mathrm{R}}\right] /\left[I_{\mathrm{L}}+I_{\mathrm{R}}\right]$, for the same film is shown. Photoluminescence intensity $I$ was recorded upon excitation with unpolarized light of $356 \mathrm{~nm}$ entering the film under normal incidence and propagating in the same direction as used for the emission detection. (b) Dissymmetry ratio $g_{\text {TRANS }}=$ $2\left(T_{\mathrm{L}}^{\mathrm{U}}-T_{\mathrm{R}}^{\mathrm{U}}\right) /\left(T_{\mathrm{L}}^{\mathrm{U}}+T_{\mathrm{R}}^{\mathrm{U}}\right)$ for thermally annealed, nonaligned films of c-PFBT with different thicknesses. The inset shows the circular selective scattering band for the $400 \mathrm{~nm}$ thick film in the near-infrared spectral range. (c) Dissymmetry ratio $g_{\text {TRANS }}=2\left(T_{\|}{ }^{\mathrm{L}}\right.$ $\left.-T_{\|}^{\mathrm{R}}\right) /\left(T_{\|}^{\mathrm{L}}+T_{\|}^{\mathrm{R}}\right)$ for aligned films of c-PFBT of different thicknesses, with $T_{\|}^{\mathrm{L}}\left(T_{\|}^{\mathrm{R}}\right)$ denoting the transmission of light that is linearly polarized in the direction parallel to the polymer chains upon entering the film and that leaves the film with left- (right-) handed circular polarization.

linearly polarized parallel to the rubbing direction of the alignment layer. This is consistent with the orientation of the transition dipole moment of the $S_{1} \leftarrow S_{0}$ transition parallel to the direction of the backbone of the polymer. The luminescence involving the reverse $S_{1} \rightarrow S_{0}$ transition is therefore expected to be initially polarized in a direction parallel to the polymer chain. However, the strong birefringence of the annealed polymer films may change the polarization of this initially linearly polarized light. Because of the cholesteric arrangement of the polymer chains, one expects some birefringence in a direction making an angle of $45^{\circ}$ with the direction of the polymer chains involved in generation of the luminescence. This particular birefringence will convert linearly polarized light into circularly polarized light and contribute to the overall circular polarization of the luminescence. In order to test this scenario experimentally, we have performed generalized ellipsometry on polymer films aligned on polyimide, focusing on the elements of the Mueller matrix involving interconversion of linear and circular polarization. Results are illustrated in Figure 4c, where we show the degree of circular polarization of light transmitted through aligned and thermally annealed films of c-PFBT of various thickness, under the condition that, on the surface of the film placed at the side of the sample where the light enters, light is linearly polarized in a direction parallel to the polymer chains. Translated to the scenario of luminescence, the resulting quantity $g_{\text {TRANS }}{ }^{\prime \prime}=2\left[T_{\|}^{\mathrm{L}}\right.$ - $\left.T_{\|}^{\mathrm{R}}\right] /\left[T_{\|}^{\mathrm{L}}+T_{\|}^{\mathrm{R}}\right]$ would correspond to the circular polarization of light being initially linearly polarized in the direction of the polymer chains at the site of light generation and having traveled through a layer of polymer of the same thickness as in the transmission measurements discussed here.

We find that $g_{\text {TRANS }}$ "is quite small for thin films and shows the expected oscillatory pattern as a function of film thickness: for films of $180 \mathrm{~nm}$ thickness we find a local maximum in the polarization with $g_{\text {TRANS }}$ " as high as -1.2 for wavelengths near $500 \mathrm{~nm}$. For as-cast films $g_{\text {TRANS }}$ "was found to be vanishingly small. The high degree of polarization in the aligned and annealed films is consisted with the transmitted light having passed through mainly a single domain within the film. Only under this condition will there be a fixed relation between the sign and direction of the birefringence and the initial linear polarization, leading to a net circular polarization. If domains in the film would be much smaller such that the transmitted light would visit several domains on its path through the film, then the induced net circular polarization is expected to cancel out. We do not have a direct measurement of how many domains light crosses in the nonaligned annealed films that we used in our OLEDs. In principle birefringence could still have a nonnegligible effect on the polarization of light generated in nonaligned films. We conclude that both circular selective scattering and birefringence could contribute to the strong CPEL that we observe in OLEDs. A quantitative assessment of the contribution of the two mechanisms is beyond the scope of the present work.

To further investigate the impact on CPEL of the disorder present in our multidomain emitting layers, we have used a model that was developed by Dmitrienko and Belyakov for transmission of light through an imperfect cholesteric liquid crystal. $^{25}$ Here we used their model to study the effect of the distribution in size and orientation of the cholesteric domains on the circular polarization of EL coming out of the OLED in the normal direction with respect to the substrate. The model considers selective reflection events at individual domains; light waves reflected from or transmitted through different domains are considered to superimpose incoherently, that is with random phases. For the film as a whole, the multiple incoherent reflections amount to scattering of light. Reflection at an individual domain of light with the same handedness as that of the cholesteric is described by

$$
r(\alpha)=B \frac{1-\cos (\alpha k h)}{\alpha^{2} k h}
$$

where

$$
B=\frac{\delta^{2}}{8 \sin ^{2} \theta}
$$

and

$$
\alpha(k)=\frac{\left(\frac{2 \pi}{p}-2 k \sin \theta\right)}{k}
$$


a)

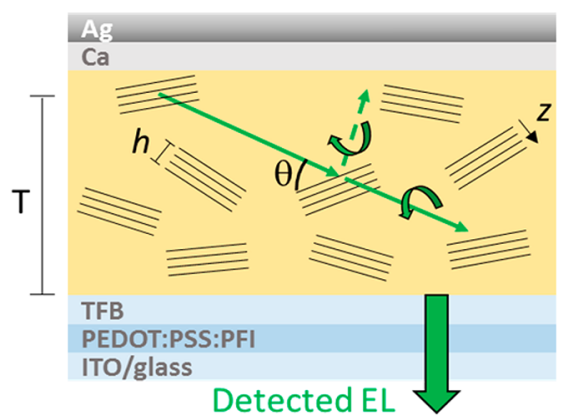

c)

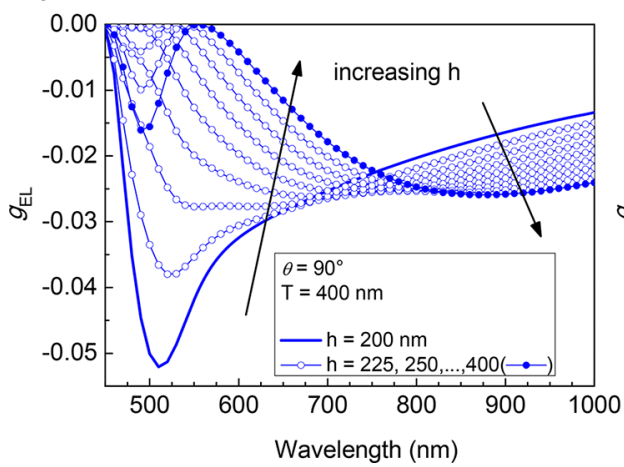

b)

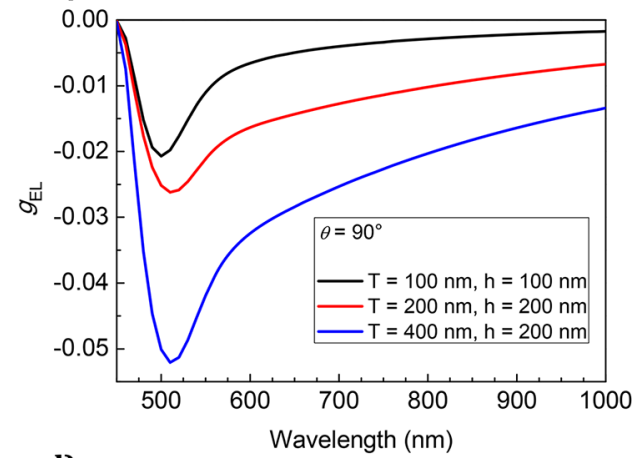

d)

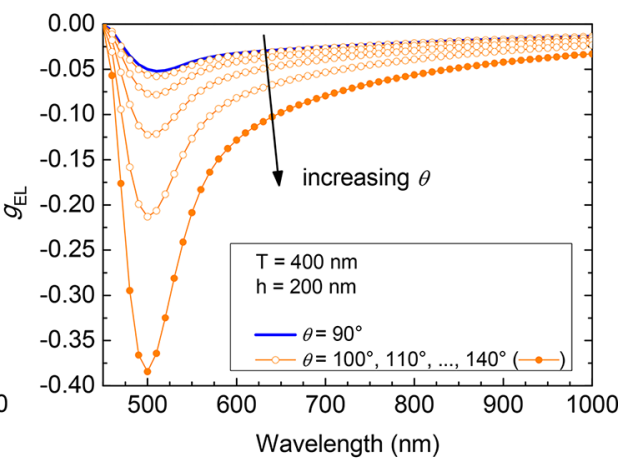

Figure 5. (a) Schematic representation of the polycrystalline cholesteric film in the OLED stack (thicknesses are not to scale). Some of the parameters used in the model are shown; the cholesteric domains are represented by groups of black lines, with $z$ being the direction of the cholesteric helical axis. The direction of detection of electroluminescence (EL) with respect to the substrate is also shown. (b) Calculated $g_{\mathrm{EL}}$ for different values of $T$ and $h$. Panels (c) and (d) show the effect of varying $h$ and $\theta$, respectively, on the calculated $g_{\mathrm{EL}}$ for $T=400 \mathrm{~nm}$. All calculations were performed using a cholesteric pitch of $600 \mathrm{~nm}$.

In eq $3, \delta$ is defined as $\delta=\left(\varepsilon_{1}-\varepsilon_{2}\right) /\left(\varepsilon_{1}+\varepsilon_{2}\right)$, where $\varepsilon_{1}$ and $\varepsilon_{2}$ are the components of the complex dielectric tensor in the plane normal to the axis of the cholesteric helix. In eq $4 p$ is the pitch of the cholesteric and $k$ the wave-vector of light in the dielectric, defined by $k=\left(\frac{2 \pi}{\lambda}\right) \bar{\varepsilon}^{1 / 2}$, where $\lambda$ is the wavelength of light in the vacuum and $\bar{\varepsilon}$ is the average dielectric function. In eq $2 h$ is the thickness of the cholesteric domain, while in eq $3 \theta$ is the angle at which light impinges on the domain. The polarization of light emerging out of the OLED depends also on the total thickness $T$ of the film from the site of emission to the interface with the transparent electrode. $T, h$, and $\theta$ are schematically represented in Figure 5a. The components $\varepsilon_{1}$ and $\varepsilon_{2}$ of the dielectric tensor were measured by ellipsometry on a thin film $(23 \mathrm{~nm})$ on quartz. The dependence of $\delta^{2}$ on wavelength obtained from the measured components of the dielectric tensor is shown in Figure S3. As mentioned above, the cholesteric pitch in our films was measured to be $600 \mathrm{~nm}$.

$B$ is almost constant at long wavelengths away from resonance, while it increases sharply approaching the onset of light absorption, due to the increase in $\delta^{2}$ (Figure S3). The range of wavelengths close to the onset of absorption is particularly relevant in our case, since it is where the peak of electroluminescence occurs. The term $\frac{1-\cos (\alpha k h)}{\alpha^{2} k h}$ in eq 2 takes into account the Bragg-type circular selective reflection of light on a single cholesteric domain.

Following the formalism of ref 25 we calculate the dissymmetry factor $g_{\mathrm{EL}}$ of the EL emitted along the direction normal to the substrate (Figure 5a) using eq 1 and the following expression for $\mathrm{EL}_{\mathrm{L}}$ and $\mathrm{EL}_{\mathrm{R}}$ :

$$
\begin{aligned}
\mathrm{EL}_{\mathrm{L}} & =\frac{m}{\left(\mu+\mu_{\mathrm{d}}\right) \sinh (m T)+m \cosh (m T)} \\
\mathrm{EL}_{\mathrm{R}} & =\mathrm{e}^{-\mu T}
\end{aligned}
$$

where $m=\left(\mu^{2}+2 \mu \mu_{\mathrm{d}}\right)^{1 / 2}, \mu$ is the linear absorption coefficient, and $\mu_{\mathrm{d}}=4 \mathrm{kr}$, with $r$ defined in eq 2 and $k$ the wave-vector of light in the dielectric.

We consider the limiting case in which light emission occurs with linear polarization and close to the cathode, with light traversing the whole emitting layer before being emitted. Figure $5 \mathrm{~b}$ shows the calculated absolute dissymmetry factor $g_{\mathrm{EL}}$ as a function of wavelength and film thickness, using $\theta=90^{\circ}$. The simulated dependence of $g_{\mathrm{EL}}$ on film thickness is in agreement with the experiment (Figure 2). The spectral dependence is also well reproduced, with the calculated $g_{\mathrm{EL}}$ peaking at $500 \mathrm{~nm}$ and decreasing monotonically with increasing wavelength, as observed in the experiments on the thinner (100 and $200 \mathrm{~nm}$ ) OLEDs. We also note that modeling successfully reproduces the red-shift of the peak of $g_{\mathrm{EL}}$ that was observed in OLEDs with increasing layer thickness (Figure 2a).

In Figure $5 b, g_{\mathrm{EL}}$ for the thickest film $(400 \mathrm{~nm})$ is calculated using $200 \mathrm{~nm}$ for the thickness of the cholesteric crystals; we show in Figure 5c that when the cholesteric crystal thickness is increased, the spectrum changes drastically, with a broad band arising and shifting to long wavelengths, peaking at $880 \mathrm{~nm}$ in the case of $h=400 \mathrm{~nm}$ (i.e., the total thickness of the film). The new band in the simulations is a consequence of the circular selective reflection of light at the individual domains (term $\frac{1-\cos (\alpha k h)}{\alpha^{2} k h}$ in eq 2). On the basis of the results in Figure 5c, we conclude that the band peaked at 700-750 nm observed in the 
$400 \mathrm{~nm}$ thick OLED (Figure 2a) indicates the existence of cholesteric domains with a range of thicknesses up to the full thickness of the emitting layer. This is also consistent with the results presented in Figure $4 \mathrm{~b}$. The calculated values of $g_{\mathrm{EL}}$ are 1 order of magnitude smaller than the experimental ones. However, when the angle of incidence of light on the single cholesteric crystals is increased, we obtain much larger values, closer to the range of the experimental values (Figure 5d). Large angles of incidence are realistic, considering the orientational disorder observed in AFM (Figure 3d).

Note that here we have shown the effect of individual parameters $(\theta, h, T)$ on the calculated $g_{\mathrm{EL}}$ : we expect the measured polarization to depend on an average over these parameters, which would take into account the profile of the recombination zone (average over a distribution of values of $T$ ), the distribution of angles of incidence $(\theta)$, and the distribution of thicknesses of the cholesteric domains inside the film $(h)$. For example, averaging over a distribution of cholesteric domain thicknesses $h$ would result in a broad spectrum of $g_{\mathrm{EL}}$ as can be appreciated from Figure $5 c$, and would explain the broad $g_{\mathrm{EL}}$ spectrum observed experimentally in the $400 \mathrm{~nm}$ thick OLED (Figure 2a). We do not have a direct measurement of the exact distribution of domain size and orientation in the films, which would be necessary for a more quantitative treatment. Furthermore, here we have not included waveguiding of EL in the emitting layer and reflection at the back metal electrode, which could also impact the spectral shape and intensity of CPEL.

In Figure 2a we have shown that $g_{\mathrm{EL}}$ goes from -0.6 to -0.8 when passing from $25 \mathrm{~V}$ constant voltage bias to $35 \mathrm{~V}$ pulsed bias $(1 \mu \mathrm{s})$. We interpret the increase in polarization as related to the variation of the recombination with bias: due to the asymmetry between hole (barrier limited) and electron (ohmic) injection into the emitting layer, the recombination zone is expected to be close to the transparent electrode; when the voltage is increased, the recombination zone extends deeper in the emitting layer, with the results that EL traverses more dielectric and the polarization increases. We found that the strongest polarization is achieved with short bias pulses, while $g_{\mathrm{EL}}$ decreases when the length of the pulses at $35 \mathrm{~V}$ is increased (Figure S2): this effect is possibly due to local heating affecting the dielectric function of the emitting layer. Heating is expected to be non-negligible at the high voltages applied on thick layers and is likely to be stronger when using long pulses or constant voltage. Our results suggest that the standard OLED structure used in this work limits the highest $g_{\mathrm{EL}}$ that is possible to obtain for a given thickness of the emitting layer, due to the unfavorable position of the recombination zone close to the transparent anode. Tuning the OLED structure in order to have the recombination zone farther from the transparent contact will allow high $g_{\mathrm{EL}}$ at lower driving voltages. The position of the recombination zone could be varied for example by choice of transport layers. In general, the rather large thickness of the cholesteric emitting layer required by our nonlocal approach to strong CPEL presents a challenge to achieving high-performance devices: to reach substantial charge injection and light emission, high driving voltages are required in the thick structures. This in turn hinders power efficiency, as can be seen in Figure S1. This limitation could be overcome by improving charge injection; Kabra et al. have previously demonstrated that ohmic hole injection in inverted-structure polymer LEDs allows the use of thick emitting layers while maintaining high power efficiency. ${ }^{26}$ We believe that if efficient charge injection is obtained in concert with optimal position of the recombination zone far from the transparent anode, strong CPEL and high power efficiency can be achieved simultaneously.

It is also worth recalling here the results by Zinna et al. in OLEDs based on chiral lanthanide complexes, where EL is strongly polarized at the site of emission (i.e., locally): ${ }^{9}$ in their study it was found that to maximize $g_{\mathrm{EL}}$ the recombination zone has to be positioned as close as possible to the transparent anode, to minimize the contribution of light which has switched to opposite polarization handedness after reflection at the metallic back electrode. It is therefore interesting to note how different mechanisms of CPEL generation translate into different requirements for the position of the recombination zone.

Finally, we show on a $100 \mathrm{~nm}$ thick c-PFBT OLED that circular polarization can be switched ON and OFF when short, high-voltage pulses are applied. The short pulses allow us to use a large range of applied voltages without damaging the OLEDs. Figure 6 shows $g_{\mathrm{EL}}$ in two different cases: $6 \mathrm{~V}, 10 \mu$ s long square

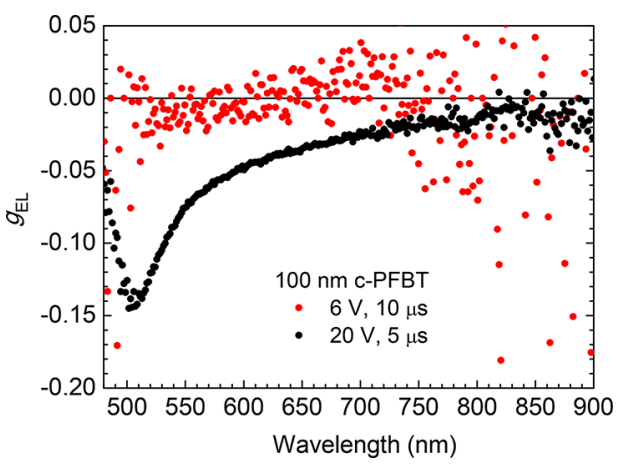

Figure 6. Dissymmetry factor of electroluminescence $g_{\mathrm{EL}}$ of an OLED comprising a $100 \mathrm{~nm}$ thick c-PFBT, measured under pulsedvoltage excitation at $6 \mathrm{~V}, 10 \mu \mathrm{s}$ (red symbols) and $20 \mathrm{~V}, 5 \mu \mathrm{s}$ (black symbols). A repetition rate of $1 \mathrm{kHz}$ was used in both cases.

pulses and $20 \mathrm{~V}, 5 \mu \mathrm{s}$ long square pulses. The repetition rate was $1 \mathrm{kHz}$ in both cases. We observe that no polarization is present when $6 \mathrm{~V}$ pulses are applied, while $g_{\mathrm{EL}}=-0.15$ at 500 $\mathrm{nm}$ is achieved with $20 \mathrm{~V}$ pulses. Similarly to the case of the 400 $\mathrm{nm}$ thick layer described above, we interpret this observation in terms of the recombination zone depth in the emitting layer: at $6 \mathrm{~V}$ and with a $10 \mu$ s pulse the recombination zone is expected to be narrow and close to the transparent electrode; under these conditions the actual dielectric thickness that EL travels across is close to zero and virtually no polarization can occur. At $20 \mathrm{~V}$, instead, there is enough electric field across the cPFBT layer to extend the recombination zone deeper, toward the back electrode. Hence a relatively large value of $g_{\mathrm{EL}}$ is achieved, ca. 2-fold higher (at $500 \mathrm{~nm}$ ) than the one recorded when using a $10 \mathrm{~V}$ constant bias (Figure 2a).

\section{CONCLUSIONS}

In conclusion, in this work we have shown very strong circular polarization of electroluminescence from single-layer, solutionprocessed polymer OLEDs based on a chiral enantiomerically pure polyfluorene copolymer as emitting material; when using a thermally annealed and thick $(400 \mathrm{~nm})$ polymer emitting layer, we recorded up to $40 \%$ excess of right-handed circular polarization under pulsed voltage bias operation $\left(g_{\mathrm{EL}}=-0.8\right)$ and $30 \%\left(g_{\mathrm{EL}}=-0.6\right)$ under constant voltage bias. These are 
between the highest values of CPEL reported for OLEDs and the highest achieved without the use of chiral dopants or alignment layers. We also showed that CPEL can be controlled electrically by varying the OLED operation mode, adding a further functionality to these devices.

We find that thermal annealing produces cholesteric films with domains having orientational and size disorder throughout the sample. The disordered, multidomain cholesteric films show no appreciable circular selective reflection. Yet domains are large enough to give rise to circular selective scattering and linear birefringence that is correlated in the direction of the initial linear polarization of the luminescence photon. Experimental determination of the Mueller matrix of the films shows efficient conversion of linearly polarized light into circularly polarized radiation. We conclude that the high levels of CPEL measured in our devices arise mainly after the photon has been generated during the passage of the photon through the film in the outward direction. The genesis of circular polarization involves selective scattering and birefringence.

We believe that the approach to CPEL based on nonlocal effects (circular selective scattering and birefringence) in multidomain cholesterics is very promising because of the ease of fabrication, only requiring thermal annealing. Another crucial advantage is that at wavelengths close to resonance, where most electroluminescence from the polymer occurs, high polarization can be achieved due to the strong dielectric anisotropy: this might not always be possible in a monodomain cholesteric film, depending on the pitch length and the resulting Bragg reflection conditions. Furthermore, we show that CPEL in our devices is broad-band, another aspect that is generally difficult to obtain if relying only on Bragg reflection in a perfect, monodomain cholesteric. Finally, we note that because the cholesteric domains are scattering centers, a beneficial impact on the out-coupling of EL in the direction normal to the OLED substrate might be expected.

Our work demonstrates a facile and generally applicable fabrication route to obtain OLEDs with high levels of circular polarization; we foresee that $100 \%$ polarized broad-band CPEL can be achieved in OLEDs with thick (a few hundreds of nanometers) multidomain cholesteric emitting layers: this will involve tuning charge mobility and injection, in order for the recombination zone to be located far from the transparent contact, already at low driving voltage. More generally, we demonstrate that chirally substituted conjugated polymers can combine semiconducting and photonic functionalities: this makes them an interesting class of materials for advanced manipulation of light in optoelectronic devices.

\section{METHODS}

Synthesis of c-PFBT. c-PFBT was synthesized ${ }^{19}$ and further purified to remove palladium catalyst according to reported procedures. $^{27,28}$

2,7-Bis(pinacoyl)-9,9-bis $((S)$-3,7-dimethyloctyl)fluorene boronic ester $(616 \mathrm{mg}, 0.88 \mathrm{mmol}, 1.0$ equiv), 4,7-dibromobenzothiadiazole (259 mg, $0.88 \mathrm{mmol}, 1.0$ equiv), and oven-dried $\left(140{ }^{\circ} \mathrm{C}\right)$ sodium carbonate (981 mg, $9.25 \mathrm{mmol}, 10.5$ equiv) were taken in a threenecked round-bottom flask (RBF, $100 \mathrm{~mL}$ ) fitted with a reflux condenser and an argon inlet. The flask was evacuated and backfilled with argon three times. Tetrakis(triphenylphosphine)palladium $(0)$ catalyst ( $51 \mathrm{mg}, 0,044 \mathrm{mmol}, 0.05$ equiv) weighed under an argon atmosphere was added to the RBF under positive argon pressure. The flask was again evacuated and backfilled with argon three times. A 2:1 $(\mathrm{v} / \mathrm{v})$ mixture of dioxane/water $(25 \mathrm{~mL})$ was degassed by three cycles of freeze-pump-thaw and added to the RBF, and the reaction mixture was heated to $95{ }^{\circ} \mathrm{C}$ (oil bath) for 3 days. Then the endcapper, 2-pinacoyl-9,9-bis $((S)$-3,7-dimethyloctyl)fluorene boronic ester ( $101 \mathrm{mg}, 0.17 \mathrm{mmol}, 0.2$ equiv), dissolved in dioxane (degassed by three cycles of freeze-pump-thaw, $6 \mathrm{~mL}$ ) was added to the RBF, and the reaction was continued overnight. The reaction mixture was allowed to cool to room temperature and extracted with chloroform and $1 \mathrm{M} \mathrm{HCl}$. The organic layer was washed with saturated $\mathrm{NaHCO}_{3}$ and finally with brine. The organic layer was dried over sodium sulfate and concentrated to about $150 \mathrm{~mL}$.

Purification. To the organic layer $\left(\mathrm{CHCl}_{3}, 150 \mathrm{~mL}\right)$ was added a $28-30 \%$ aqueous ammonia solution $(150 \mathrm{~mL})$, and the mixture was refluxed (oil bath at $85{ }^{\circ} \mathrm{C}$ ) for $3 \mathrm{~h}$ and then stirred overnight at room temperature. The next day, the solution was transferred to a separating funnel and extracted with excess water. To the collected organic layer was added ethylenediaminetetraacetic acid disodium salt dihydrate $(1.2 \mathrm{~g})$ and further stirred at room temperature overnight. After $15 \mathrm{~h}$, the organic layer was extracted with water, dried (over sodium sulfate), and evaporated to about $10-15 \mathrm{~mL}$. To this concentrated solution was added a palladium scavenger agent, diethyldithiocarbamic acid diethylammonium salt $(25 \mathrm{mg})$, and the mixture was stirred under dark and in an argon atmosphere at room temperature for $8 \mathrm{~h}$. Immediately after addition of the scavenger, the dark-colored solution turned orange. After $8 \mathrm{~h}$, the solution was poured into cold methanol $(250 \mathrm{~mL})$, and the yellow precipitate was collected by suction filtration and dried under vacuum at $60^{\circ} \mathrm{C}$ overnight. The precipitate was then subjected to Soxhlet extraction with first acetone and finally with chloroform (oil bath at $85{ }^{\circ} \mathrm{C}$ ) for $24 \mathrm{~h}$ each. The chloroform was concentrated to about $10 \mathrm{~mL}$ and poured into cold methanol (300 $\mathrm{mL}$ ). The precipitate was collected by suction filtration and dried under vacuum at $60{ }^{\circ} \mathrm{C}$ for $24 \mathrm{~h}$ to obtain a yellow solid ( $440 \mathrm{mg}, 82 \%$ yield). ${ }^{1} \mathrm{H}$ NMR (400 MHz, $\left.\mathrm{CDCl}_{3}\right): \delta=8.11-8.09(\mathrm{~m}, 2 \mathrm{H}), 8.02-$ $7.86(\mathrm{~m}, 6 \mathrm{H}), 2.16(\mathrm{~m}, 4 \mathrm{H}), 1.44-1.36(\mathrm{~m}, 2 \mathrm{H}), 1.30-0.85(\mathrm{~m}, 19 \mathrm{H})$, $0.85-0.6 \mathrm{ppm}(\mathrm{m}, 24 \mathrm{H}) .{ }^{13} \mathrm{C}$ NMR $\left(100 \mathrm{MHz}, \mathrm{CDCl}_{3}\right): \delta=154.55$, 151.82 , 141.14, 136.66, 133.82, 128.48, 128.08, 124.12, 120.17, 55.42, $39.42,37.68,36.86,33.18,30.94,28.06,24.88,22.79,22.69,19.80$ ppm. Both ${ }^{1} \mathrm{H}$ and ${ }^{13} \mathrm{C}$ NMR spectra are in agreement with those reported in the literature. ${ }^{20}$ GPC (PS standard, THF): apparent $M_{n}$ $15.36 \mathrm{~kg} \mathrm{~mol}^{-1}, M_{\mathrm{w}} 31.77 \mathrm{~kg} \mathrm{~mol}^{-1}$; PDI $\left(M_{\mathrm{w}} / M_{\mathrm{n}}\right)=2.06$

OLED Fabrication and Characterization. OLEDs were fabricated by spin coating in air (5000 rpm, $2000 \mathrm{rpm} / \mathrm{s}$ acceleration) a mixture of PEDOT:PSS (poly(ethylenedioxythiophene):poly(styrenesulfonate), Clevios P VP Al4083) and PFI (Nafion perfluorinated resin solution, $5 \mathrm{wt} \%$ in lower aliphatic alcohols and water, containing $15-20 \%$ water, Sigma-Aldrich) onto precleaned, patterned indium tin oxide (ITO) substrates (15 $\Omega$ per square, Colorado Concept Coatings). PEDOT:PSS:PFI was prepared in the weight ratio 1:6:11.2. The PEDOT:PSS:PFI layer was annealed at 200 ${ }^{\circ} \mathrm{C}$ for 20 min under a $\mathrm{N}_{2}$ atmosphere. Subsequently, a layer of TFB $\left(M_{\mathrm{p}}=119 \mathrm{~kg} / \mathrm{mol}, M_{\mathrm{n}}=60 \mathrm{~kg} / \mathrm{mol}\right)$ was spin coated $(2000 \mathrm{rpm}, 2000$ $\mathrm{rpm} / \mathrm{s}$ acceleration) using an $8 \mathrm{mg} / \mathrm{mL}$ solution in toluene. The TFB layer was thermally annealed at $180{ }^{\circ} \mathrm{C}$ for $20 \mathrm{~min}$ under a $\mathrm{N}_{2}$ atmosphere before depositing the c-PFBT layer. The c-PFBT layer was spin coated at $2000 \mathrm{rpm}, 2000 \mathrm{rpm} / \mathrm{s}$ acceleration, with concentration varied between 15 and $40 \mathrm{mg} / \mathrm{mL}$ in toluene to achieve different thicknesses. At this point the stack was further annealed at $240{ }^{\circ} \mathrm{C}$ for $15 \mathrm{~min}$ and rapidly cooled afterward. Finally, $10 \mathrm{~nm}$ of calcium and 80 $\mathrm{nm}$ of silver were thermally evaporated at a pressure of $10^{-6} \mathrm{mbar}$. The devices were encapsulated to limit degradation during the measurements. The OLEDs had an active area of $4.5 \mathrm{~mm}^{2}$. Currentluminance-voltage curves were recorded in a setup consisting of a Keithley 2400 source meter and a calibrated silicon photodiode. The thickness of the c-PFBT layer was measured with a Bruker Dektak profilometer.

Measurement of Circularly Polarized Electroluminescence. Electroluminescence spectra were recorded by using an Andor Shamrock spectrometer (model SR-303i-B), equipped with a silicon CCD (model iDUS DU420A). The EL spectra were corrected for the response of the spectrometer and detector. Circular polarization was measured by placing a broad-band $\lambda / 4$ waveplate (Thorlabs, AQWP05M-600) between the OLED and a linear polarizer. 
Measurements were conducted using the direction of EL normal to the OLED substrate, the $\lambda / 4$ waveplate, and the linear polarizer. $\mathrm{EL}_{\mathrm{L}}$ and $\mathrm{EL}_{\mathrm{R}}$ were measured by rotating the fast axis of the $\lambda / 4$ waveplate at an angle of $-45^{\circ}\left(+45^{\circ}\right)$ with respect to the polarization axis of the linear polarizer, respectively. The linear polarizer was maintained in a fixed position to avoid artifacts arising from the sensitivity of the monochromator to linear polarization. A 2400 Keithley source meter was used to drive the OLEDs in constant voltage bias. A function generator (Hewlett-Packard, model 8116A) coupled with a home-built high-voltage source was used for the pulsed-voltage experiments. The voltage pulses were obtained from a square wave at a repetition rate of $1 \mathrm{kHz}$, with the duration of the high-voltage state varied between 1 and $250 \mu \mathrm{s}$ and the $0 \mathrm{~V}$ state maintained for the rest of the cycle. All the experiments were conducted at room temperature.

Atomic Force Microscopy. AFM was carried out using an Asylum Research MFP-3D mounted on an antivibration stage. Silicone probes manufactured by NanoSensors (model PPP-NCSTR-50) with a tip height of $10-15 \mu \mathrm{m}$ and radius of $<10 \mathrm{~nm}$ were used for AC tappingmode measurement. A scan rate of $0.8 \mathrm{~Hz}$ and pixel size of $512 \times 512$ resolution were used. The images were subjected to first-order flattening using Gwyddion (v. 2.48) software.

Polarized Optical Microscopy. POM images were acquired on a Leica DM $2700 \mathrm{M}$ optical microscope equipped with cross polarizers.

Circular Dichroism. Circular dichroism (CD) measurements on a film with thickness $d<140 \mathrm{~nm}$ were done on a JASCO J-815 spectrometer using a scan speed of $100 \mathrm{~nm} \mathrm{~min}{ }^{-1}$, data pitch of 0.1 $\mathrm{nm}$, response time of $1 \mathrm{~s}$, and bandwidth of 1.71. Linear dichroism (LD) was measured on all the samples, and no significant LD was observed for the samples studied. CD results on thick, $d>140 \mathrm{~nm}$, films were obtained from generalized ellipsometry with a Woollam WVASE unit. The $(1,4)$ element of the Mueller matrix in transmission geometry equals $-1 / 2$ times the dissymmetry ratio for transmission, $g_{\text {TRANS. }}$ Combining the Mueller matrix determination with independent measurement of the transmission, the extinctions $E_{\mathrm{L}}^{\mathrm{U}}$ and $E_{\mathrm{R}}^{\mathrm{U}}$ can be calculated in a straightforward manner. The $(2,4)$ element of the Mueller matrix measured on an aligned film with alignment layer on the detector side and rubbing direction equals $1 / 2$ times $g_{\text {TRANS }}$ ".

Sample Preparation for Optical and Microscopy Studies. Thin films were spin-coated from solution of c-PFBT with varying concentrations (from 5 to $50 \mathrm{mg} / \mathrm{mL}$ ) to obtain a range of thicknesses. All films were spin-coated from toluene, with the exception of thin films for circular dichroism (Figure 3a) and aligned films (Figure 4c), which were spin-coated from a solution of c-PFBT in 9:1 (v/v) chloroform/chlorobenzene. The solutions were spin-coated (2000 $\mathrm{rpm}, 60 \mathrm{~s}$ ) on cleaned glass or fused silica substrates. All annealed samples were annealed for 15 min under a nitrogen atmosphere (glovebox). The film thicknesses of all films were measured using a Dektak 150 surface profiler.

Polyimide Alignment Layer. Glass slides of $2.5 \mathrm{~cm} \times 2.5 \mathrm{~cm}$ were cleaned by sonication for $10 \mathrm{~min}$ each with acetone and isopropyl alcohol. The glass slides were then etched in a UV-ozone photoreactor (PR-100) for $30 \mathrm{~min}$. Polyimide (AL 1051) was spin-coated on the etched glass slides (5000 rpm, $40 \mathrm{~s}, 500 \mathrm{rpm}$ acceleration) and then annealed at $180{ }^{\circ} \mathrm{C}$ for $90 \mathrm{~min}$. The polyimide facing side was rubbed on a velvet cloth to induce planar alignment.

Circular Polarization of Photoluminescence. Measurements were done using a home-built setup employing a photoelastic modulator and a 16-channel photomultiplier array involving a photon counting detection scheme. Excitation light of $365 \mathrm{~nm}$ was selected from a $\mathrm{Hg}$ lamp using appropriate interference and bandpass filters. The excitation light was depolarized by passing it through a bundle of optical fibers. The direction of the excitation was normal to the surface of the film and in line with the direction of emission collection.

Ellipsometry. Generalized ellipsometry measurements were carried out with the Woollam WVASE ellipsometer using the general Mueller matrix measurement protocol.

\section{ASSOCIATED CONTENT}

\section{Supporting Information}

The Supporting Information is available free of charge on the ACS Publications website at DOI: 10.1021/acsnano.7b07390.

Current-luminance-voltage characteristics, $g_{\mathrm{EL}}$ as a function of operation mode, experimental values of $\delta^{2}$ (PDF)

\section{AUTHOR INFORMATION}

\section{Corresponding Author}

*E-mail (D. Di Nuzzo): dd467@cam.ac.uk.

ORCID

Daniele Di Nuzzo: 0000-0002-4462-9068

Chidambar Kulkarni: 0000-0001-8342-9256

Stefan C. J. Meskers: 0000-0001-9236-591X

Ron Naaman: 0000-0003-1910-366X

E. W. Meijer: 0000-0003-4126-7492

Richard H. Friend: 0000-0001-6565-6308

\section{Notes}

The authors declare no competing financial interest.

\section{ACKNOWLEDGMENTS}

D.D.N. and R.H.F. acknowledge financial support from the Engineering and Physical Sciences Research Council of the UK (EPSRC). C.K. and E.W.M. acknowledge financial support from the Dutch Ministry of Education, Culture and Science (Gravity program 024.001.035). C.K. is grateful for financial support from a Marie Skłodowska-Curie (704830) postdoctoral fellowship.

\section{REFERENCES}

(1) Hövel, S.; Gerhardt, N. C.; Hofmann, M. R.; Lo, F.-Y.; Reuter, D.; Wieck, A. D.; Schuster, E.; Keune, W.; Wende, H.; Petracic, O.; Westerholt, K. Electrical Detection of Photoinduced Spins Both at Room Temperature and in Remanence. Appl. Phys. Lett. 2008, 92, 242102.

(2) Farshchi, R.; Ramsteiner, M.; Herfort, J.; Tahraoui, A.; Grahn, H. T. Optical Communication of Spin Information Between Light Emitting Diodes. Appl. Phys. Lett. 2011, 98, 162508.

(3) Wang, C. S.; Fei, H. S.; Qiu, Y.; Yang, Y. Q.; Wei, Z. Q. Photoinduced Birefringence and Reversible Optical Storage in LiquidCrystalline Azobenzene Side-Chain Polymers. Appl. Phys. Lett. 1999, $74,19-21$.

(4) Liu, C.; Yang, D.; Jin, Q.; Zhang, L.; Liu, M. A Chiroptical Logic Circuit Based on Self-Assembled Soft Materials Containing Amphiphilic Spiropyran. Adv. Mater. 2016, 28, 1644-1649.

(5) Novikova, T.; Pierangelo, A.; Manhas, S.; Benali, A.; Validire, P.; Gayet, B.; De Martino, A. The Origins of Polarimetric Image Contrast between Healthy and Cancerous Human Colon Tissue. Appl. Phys. Lett. 2013, 102, 241103.

(6) Kunnen, B.; Macdonald, C.; Doronin, A.; Jacques, S.; Eccles, M.; Meglinski, I. Application of Circularly Polarized Light for Non-Invasive Diagnosis of Cancerous Tissues and Turbid Tissue-like Scattering Media. J. Biophotonics 2015, 8, 317-323.

(7) McNichols, R. J.; Cote, G. L. Optical Glucose Sensing in Biological Fluids: An Overview. J. Biomed. Opt. 2000, 5, 5-16.

(8) Lee, D.-M.; Song, J.-W.; Lee, Y.-J.; Yu, C.-J.; Kim, J.-H. Control of Circularly Polarized Electroluminescence in Induced Twist Structure of Conjugate Polymer. Adv. Mater. 2017, 29, 1700907.

(9) Zinna, F.; Pasini, M.; Galeotti, F.; Botta, C.; Di Bari, L.; Giovanella, U. Design of Lanthanide-Based OLEDs with Remarkable Circularly Polarized Electroluminescence. Adv. Funct. Mater. 2017, 27, 1603719. 
(10) Brandt, J. R.; Wang, X.; Yang, Y.; Campbell, A. J.; Fuchter, M. J. Circularly Polarized Phosphorescent Electroluminescence with a High Dissymmetry Factor from PHOLEDs Based on a Platinahelicene. J. Am. Chem. Soc. 2016, 138, 9743-9746.

(11) Li, T.-Y.; Jing, Y.-M.; Liu, X.; Zhao, Y.; Shi, L.; Tang, Z.; Zheng, Y.-X.; Zuo, J.-L. Circularly Polarised Phosphorescent Photoluminescence and Electroluminescence of Iridium Complexes. Sci. Rep. 2015, $5,14912$.

(12) Han, J.; Guo, S.; Wang, J.; Wei, L.; Zhuang, Y.; Liu, S.; Zhao, Q.; Zhang, X.; Huang, W. Circularly Polarized Phosphorescent Electroluminescence from Chiral Cationic Iridium(III) Isocyanide Complexes. Adv. Opt. Mater. 2017, 5, 1700359.

(13) Peeters, E.; Christiaans, M. P. T.; Janssen, R. A. J.; Schoo, H. F. M.; Dekkers, H.; Meijer, E. W. Circularly Polarized Electroluminescence from a Polymer Light-Emitting Diode. J. Am. Chem. Soc. 1997, 119, 9909-9910.

(14) Oda, M.; Nothofer, H. G.; Lieser, G.; Scherf, U.; Meskers, S. C. J.; Neher, D. Circularly Polarized Electroluminescence from LiquidCrystalline Chiral Polyfluorenes. Adv. Mater. 2000, 12, 362-365.

(15) Geng, Y. H.; Trajkovska, A.; Culligan, S. W.; Ou, J. J.; Chen, H. M. P.; Katsis, D.; Chen, S. H. Origin of Strong Chiroptical Activities in Films of Nonafluorenes with a Varying Extent of Pendant Chirality. J. Am. Chem. Soc. 2003, 125, 14032-14038.

(16) Yang, Y.; da Costa, R. C.; Smilgies, D.-M.; Campbell, A. J.; Fuchter, M. J. Induction of Circularly Polarized Electroluminescence from an Achiral Light-Emitting Polymer via a Chiral Small-Molecule Dopant. Adv. Mater. 2013, 25, 2624-2628.

(17) Blok, P.; Dekkers, H. Discrimination Between 3-Pi-Pi-Star and 3n-Pi-Star States in Organic Molecules by Circular-Polarization of Phosphorescence. Chem. Phys. Lett. 1989, 161, 188-194.

(18) Lunkley, J. L.; Shirotani, D.; Yamanari, K.; Kaizaki, S.; Muller, G. Extraordinary Circularly Polarized Luminescence Activity Exhibited by Cesium Tetrakis(3-Heptafluoro-Butylryl-(+)-Camphorato) Eu(III) Complexes in $\mathrm{EtOH}$ and $\mathrm{CHCl} 3$ Solutions. J. Am. Chem. Soc. 2008, 130, 13814-13815.

(19) Geng, Y. H.; Trajkovska, A.; Katsis, D.; Ou, J. J.; Culligan, S. W.; Chen, S. H. Synthesis, Characterization, and Optical Properties of Monodisperse Chiral Oligofluorenes. J. Am. Chem. Soc. 2002, 124, 8337-8347.

(20) Abbel, R.; Schenning, A. P. H. J.; Meijer, E. W. Molecular Weight Optimum in the Mesoscopic Order of Chiral Fluorene (Co)Polymer Films. Macromolecules 2008, 41, 7497-7504.

(21) Lakhwani, G.; Meskers, S. C. J. Insights from Chiral Polyfluorene on the Unification of Molecular Exciton and Cholesteric Liquid Crystal Theories for Chiroptical Phenomena. J. Phys. Chem. A 2012, 116, 1121-1128.

(22) Lee, T.-W.; Chung, Y.; Kwon, O.; Park, J.-J. Self-Organized Gradient Hole Injection to Improve the Performance of Polymer Electroluminescent Devices. Adv. Funct. Mater. 2007, 17, 390-396.

(23) Kim, J. S.; Friend, R. H.; Grizzi, I.; Burroughes, J. H. Spin-Cast

Thin Semiconducting Polymer Interlayer for Improving Device Efficiency of Polymer Light-Emitting Diodes. Appl. Phys. Lett. 2005, $87,023506$.

(24) Lakhwani, G.; Meskers, S. C. J.; Janssen, R. A. J. Circular Differential Scattering of Light in Films of Chiral Polyfluorene. J. Phys. Chem. B 2007, 111, 5124-5131.

(25) Dmitrienko, V.; Belyakov, V. Contribution to the Theory of the Optical Properties of Imperfect Cholesteric Liquid Crystals. Sov. Phys. JETP 1977, 46, 362-356.

(26) Kabra, D.; Lu, L. P.; Song, M. H.; Snaith, H. J.; Friend, R. H. Efficient Single-Layer Polymer Light-Emitting Diodes. Adv. Mater. 2010, 22, 3194-3198.

(27) Bracher, C.; Yi, H.; Scarratt, N. W.; Masters, R.; Pearson, A. J.; Rodenburg, C.; Iraqi, A.; Lidzey, D. G. The Effect of Residual Palladium Catalyst on the Performance and Stability of PCDTBT:PC70BM Organic Solar Cells. Org. Electron. 2015, 27, 266-273.

(28) Patel, D. G.; Graham, K. R.; Reynolds, J. R. A Diels-Alder Crosslinkable Host Polymer for Improved PLED Performance: The
Impact on Solution Processed Doped Device and Multilayer Device Performance. J. Mater. Chem. 2012, 22, 3004-3014. 